\title{
Studies of Hyperons and Antihyperons in Nuclei
}

\author{
Josef POCHODZALLA* \\ Johannes Gutenberg-Universität Mainz, Institut für Kernphysik, D-55099 Germany \\ E-mail: pochodzadkph.uni-mainz.de
}

\section{Alexander BOTVINA}

Institute for Nuclear Research, Russian Academy of Sciences, 117312 Moscow, Russia

E-mail: a.botvinalgsi.de

\section{Alicia SANCHEZ LORENTE}

Johannes Gutenberg-Universität Mainz, Institut für Kernphysik, D-55099 Germany

E-mail: Lorentedkph.uni-mainz.de

\begin{abstract}
Stored antiproton beams at the international FAIR facility will provide unique opportunities to study hyperons as well as antihyperons in nuclear systems. Precise $\gamma$-spectroscopy of multistrange hypernuclei will serve as a laboratory for the hyperon-hyperon interaction. Exclusive hadron-antihadron pair production close to threshold can measure the potential of a antihadron relative to that of the coincident hadrons.

In the present work we explore the production of excited states in double hypernuclei following the micro-canonical break-up of an initially excited double hypernucleus which is created by the absorption and conversion of a stopped $\Xi^{-}$hyperon. Generally the formation of excited hypernuclear states relative to ground states dominates in this model. For different initial target nuclei which absorb the $\Xi^{-}$, different double hypernuclei nuclei dominate. We also compare the model predictions with the correlated pion spectra measured by the E906 collaboration.

In antiproton nucleus reactions the event-by-event transverse momentum correlations of hadronantihadron pairs produced close to threshold contain information on the difference between the nuclear potential of the hadron and the associated antihadron. For produced D-meson pairs at $6.7 \mathrm{GeV} / c$ the sensitivity of the transverse momenta correlation will probably be to small to deduce differences between the potentials for $\mathrm{D}^{+}$and $\mathrm{D}^{-}$mesons. However, for $\Xi \bar{\Xi}$ pairs produced at $2.9 \mathrm{GeV} / c$ the asymmetry is sufficiently sensitive to predicted differences between the $\Xi$ and $\bar{\Xi}$ potentials even if the momentum and density dependence of the the potential are taken into account.
\end{abstract}

XLVIII International Winter Meeting on Nuclear Physics in Memoriam of Ileana Iori 25-29 January 2010

Bormio, Italy

\footnotetext{
*Speaker.
} 


\section{Introduction}

Quantum Chromo Dynamics (QCD) is the theory of the force responsible for the binding of nucleons and nuclei and thus of a significant fraction of the ordinary matter in our universe. While the internal structure of hadrons and the spectra of their excited states are important aspects of $\mathrm{QCD}$, it is at least equally important to understand how nuclear physics emerges in a more rigorous way out of QCD and how nuclear structures - nuclei on the small scale and dense stellar objects on the large scale - are formed. In particular the role of strangeness in neutron stars has not been settled yet.

The study of hypernuclei can illuminate features that are obscured in conventional nuclear systems. The hyperon offers a selective probe of the hadronic many-body problem as it is not restricted by the Pauli principle. Since direct scattering experiments between two hyperons are impractical, the precise spectroscopy of multi-strange hypernuclei provides a unique chance to explore the hyperon-hyperon interaction. Significant progress in nuclear structure calculations in chiral effective field theory nurtures the hope that detailed information on excitation spectra of double hypernuclei will provide unique information on the hyperon-hyperon interactions. At the same time, the $\Lambda-\mathrm{N}$ and $\Lambda-\Lambda$ weak interaction can be studied by hypernuclei decays, opening a inimitable window for the four-baryon, strangeness non-conserving weak interaction.

Furthermore, the physics of strangeness in hadronic systems is a constantly developing field. It brings up new, often unexpected results, new challenges and open questions like anti-hypernuclei, kaonic nuclei, exotic hadronic states like the controversially discussed pentaquark baryons and the H-dibaryon. Based on G-parity transformation [四] Dürr and Teller predicted within an early form of a relativistic field theory a strongly attractive potential for antiprotons in nuclei [0]. First investigations of antiproton-nucleus scattering cross sections [ [3, , 4 , []] showed however disagreement

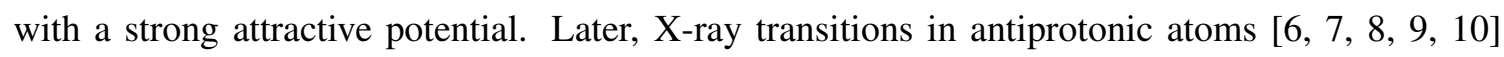
gave also hints for an attractive potential albeit with large uncertainties [प], ए2]. More comprehensive studies [[13] of antiprotonic X-rays as well as recent analyses of the production of antiprotons in reactions with heavy ions resulted in attractive real potentials in the range of about -100 to $150 \mathrm{MeV}$ [144, [15, [6]]. It is obvious that G-parity can only provide a link between the $N N$ and $N \bar{N}$ interactions for distances where meson exchange is a valid concept [ए], [18]. For distances lower than about $1 \mathrm{fm}$, quark degrees of freedom may play a decisive role. Nevertheless this consideration still indicates that a direct comparison of the interactions of baryons and antibaryons in nuclei may help to shed some light on the nature of short-range baryon-baryon forces.

\section{Production of Double Hypernuclei}

The simultaneous production and implementation of two $\Lambda$ particles into a nucleus is intricate. There is a possibility to produce multi-strange hypernuclei in heavy ion collisions via coalescence [107, [20]. The first observation of antihypernuclei by the STAR collaboration impressively illustrates the potential of this method [ [2]]. However, high resolution spectroscopy of excited states is not feasible. To produce double hypernuclei in a more 'controlled' way the conversion of a captured $\Xi^{-}$and a proton into two $\Lambda$ particles can be used. This process releases - ignoring binding energy effects - only $28 \mathrm{MeV}$. For light nuclei there exists therefore a significant probability of the order 


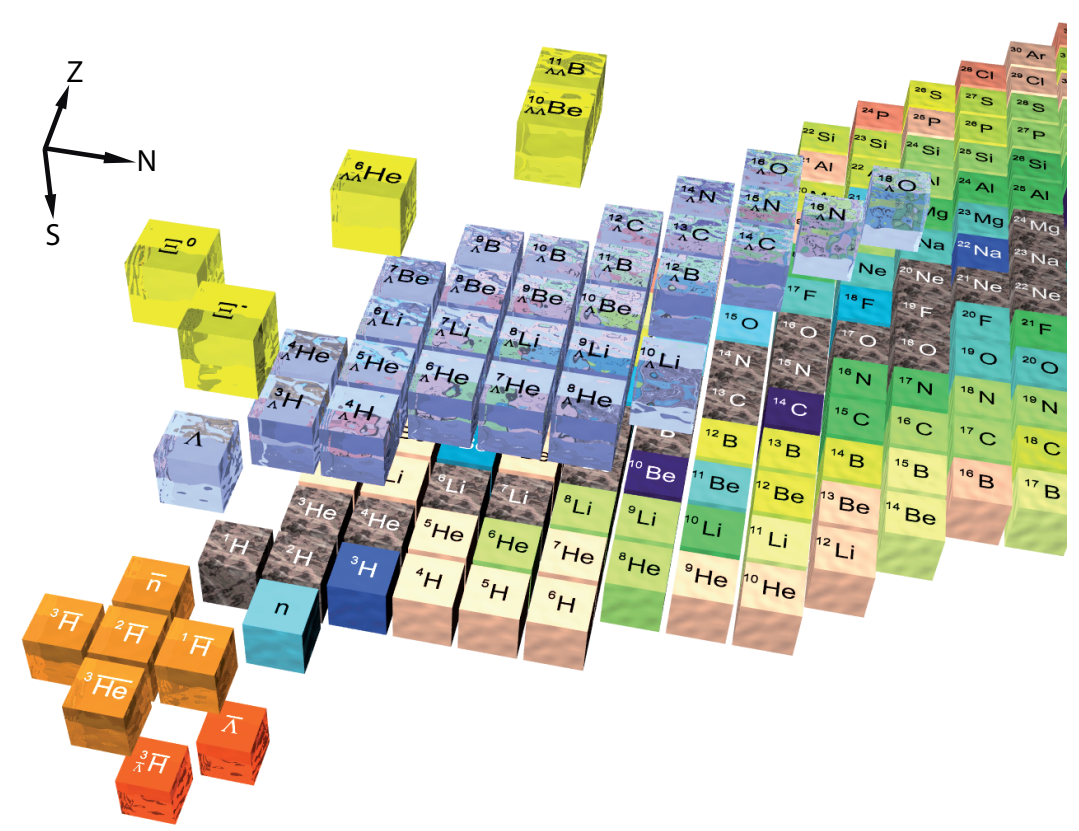

Figure 1: Present knowledge on $S=-1$ nuclei (blue) and $S=-2$ nuclei (yellow). Only very few individual events of double hypernuclei have been detected and identified so far. First antihypertritons were recently observed by the STAR collaboration [R]].

of a few percent that both $\Lambda$ hyperons are trapped in the same excited nuclear fragment [122, 223, [24, [25, [26, 27].

Unfortunately $\Xi^{-}$hyperons produced in reactions with stable hadron beams have usually rather high momenta. Therefore, direct capture of the $\Xi^{-}$in the nucleus is rather unlikely. Even in case of the $\left(\mathrm{K}^{-}, \mathrm{K}^{+}\right)$double strangeness exchange reaction, $\Xi^{-}$hyperons are produced with typical momenta of $500 \mathrm{MeV} / c$ at beam momenta around $1.8 \mathrm{GeV} / \mathrm{c}$ [24, 28]]. The advantage of this production process is that the outgoing $\mathrm{K}^{+}$can be used as a tag for the reaction. A drawback is the low kaon beam intensity and hence the need for thick primary targets. Furthermore, as a consequence of the large momentum transfer, the probability to form bound $\Xi^{-}$states directly is rather small on the level of $1 \%\left[\mathbb{Z Q},[0]\right.$ and the production of quasi-free $\Xi^{-}$dominates. Still the $\Xi^{-}$ hyperons in the quasi-free region may be absorbed into the target nucleus via a rescattering process on a nucleon which itself is knocked out of the primary nucleus. This two-step process is predicted to exceed the direct capture by more than a factor of 6 [2] ].

On the other hand most $(\sim 80 \%) \Xi^{-}$hyperons escape from the primary target nucleus in $\left(\mathrm{K}^{-}, \mathrm{K}^{+}\right)$reactions. However, in a second step, these $\Xi^{-}$hyperons can be slowed down in a dense, solid material (e.g. a nuclear emulsion) and form $\Xi^{-}$atoms [B]]. After an atomic cascade, the $\Xi$-hyperon is eventually captured by a secondary target nucleus and converted via the $\Xi^{-} p \rightarrow \Lambda \Lambda$ reaction into two $\Lambda$ hyperons. In a similar two-step process relatively low momentum $\Xi^{-}$can also be produced using antiproton beams in $\bar{p} p \rightarrow \Xi^{-} \bar{\Xi}^{+}$or $\bar{p} n \rightarrow \Xi^{-} \bar{\Xi}^{\circ}$ reactions if this reactions happens in a complex nucleus where the produced $\Xi^{-}$can re-scatter [32, [33]. The advantage as 
compared to the kaon induced reaction is that antiprotons are stable and can be retained in a storage ring. This allows a rather high luminosity even with very thin primary targets.

Because of the two-step mechanism, spectroscopic studies, based on two-body kinematics like in single hypernucleus production, cannot be performed. Spectroscopic information on double hypernuclei can therefore only be obtained via their decay products. The kinetic energies of weak decay products are sensitive to the binding energies of the two $\Lambda$ hyperons. While the double pionic decay of light double hypernuclei can be used as an effective experimental filter to reduce the background [34] the unique identification of hypernuclei groundstates only via their pionic decay is usually hampered by the limited resolution. Instead, $\gamma$-rays emitted via the sequential decay of excited double hypernuclei may provide precise information on the level structure.

\subsection{Statistical Decay of excited Doubly Strange Nuclei}

The PANDA experiment [33] which is planned at the international Facility for Antiproton and Ion Research FAIR in Darmstadt aims at the high resolution $\gamma$-ray spectroscopy of double hypernuclei [B2]. An important question is to what extent double hypernuclei in excited, particle stable states are populated following the break-up of an highly excited doubly strange nucleus which is formed after the absorption and conversion of a stopped $\Xi^{-}$. For light nuclei even a relatively small excitation energy may be comparable with their binding energy. We therefore consider the explosive decay of the excited nucleus into several smaller clusters as the principal mechanism of de-excitation.

To describe this break-up process we have developed a model [BS] which is similar to the famous Fermi model for particle production in nuclear reactions [36]. We assume that the nucleus with mass numbers $A_{0}$, charge $Z_{0}$, and the number of $\Lambda$ hyperons $H_{0}$ (here $H_{0}=2$ ) breaks-up simultaneously into cold and slightly excited fragments, which have a lifetime longer than the decay time, estimated as an order of 100-300 fm/c. In the model we consider all possible break-up channels, which satisfy the mass number, hyperon number (i.e. strangeness), charge, energy and momentum conservations, and take into account the competition between these channels.

The excitation energy of the initial, highly excited double $\Lambda$ nucleus is determined by the binding energy of the captured $\Xi^{-}$hyperon. Unfortunately, still very little is established experimentally on the interaction of $\Xi^{-}$hyperons with nuclei. Various data suggest a nuclear potential well depth around $20 \mathrm{MeV}$ (see e.g. [B], [0]]). Calculations of light $\Xi$ atoms [3] predict that the conversion of the captured $\Xi^{-}$from excited states with correspondingly small binding energies dominates. In a nuclear emulsion experiment a $\Xi^{-}$capture at rest with two single hyperfragments has been observed [38] which was interpreted as $\Xi^{-}+{ }^{12} \mathrm{C} \rightarrow{ }_{\Lambda}^{4} \mathrm{H}+{ }_{\Lambda}^{9} \mathrm{Be}$ reaction. The deduced binding energy of the $\Xi^{-}$varied between $0.62 \mathrm{MeV}$ and $3.70 \mathrm{MeV}$, depending whether only one out of the two hyperfragments or both fragments were produced in an excited particle stable state. In order to take into account the uncertainties of the excitation energy of the converted $\Xi^{-}$-states, the calculations were performed for a range of energies $0 \leq B_{\Xi} \leq E_{\max }$, constructing in this way the excitation functions for the production of hypernuclei.

Fig. $\square$ shows as an example the production of ground (g.s.) and excited (ex.s.) states of single + one free $\Lambda$ (SHP), twin (THP) and double (DHP) hypernuclei in case of a ${ }^{12} \mathrm{C}$ target as a function of the assumed $\Xi^{-}$binding energy. With increasing $\Xi^{-}$binding energy the excitation energy of the excited primary ${ }_{\Lambda \Lambda}^{13} \mathrm{~B}^{*}$ nucleus decreases from left to right from about $40 \mathrm{MeV}$ to $15 \mathrm{MeV}$. For all 


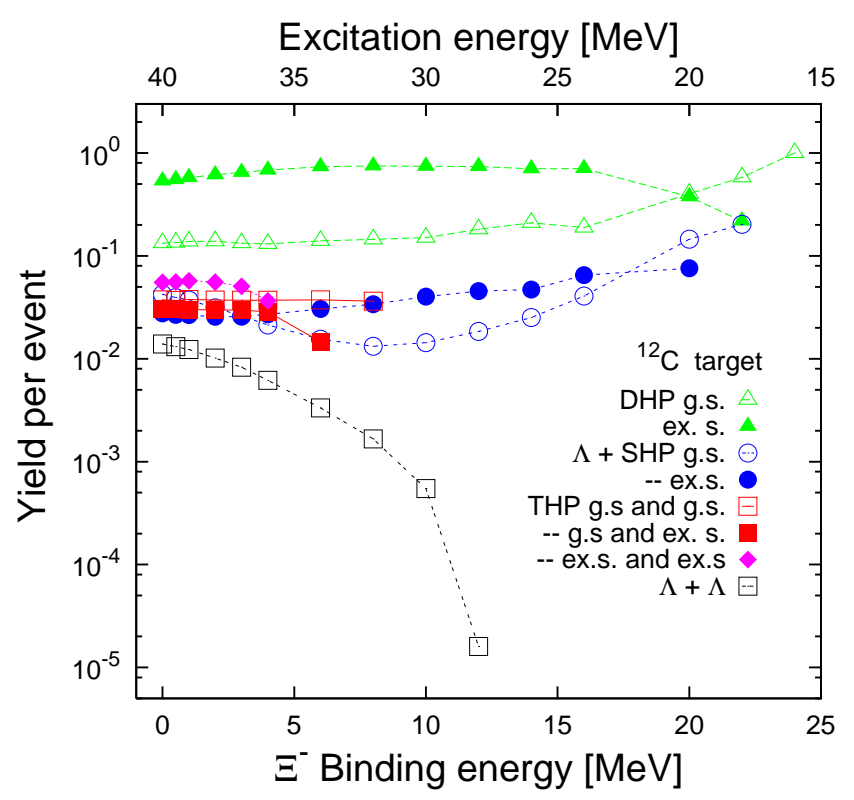

Figure 2: Predicted production probability of ground (g.s.) and excited states (ex.s.) in one single (SHP), twin (THP) and double hypernuclei (DHP) after the capture of a $\Xi^{-}$in a ${ }^{12} \mathrm{C}$ nucleus and its conversion into two $\Lambda$ hyperons. The lower and upper scale shows the binding energy of the captured $\Xi^{-}$and the excitation energy of the initial ${ }_{\Lambda \Lambda}^{13} \mathrm{~B}$ nucleus, respectively (from Ref. [B]]]).

excitation energies above $20 \mathrm{MeV}$ the production of excited double hypernuclei dominates (green triangles). This can be traced back to the opening of several thresholds for various excited double hypernuclei already at moderate excitation energies. Only for small binding energies and hence large excitation energies the production of single and twin hypernuclei is significant $(\sim 10 \%)$. The non-monotonic behaviour for single hypernucleus + one free $\Lambda$ production reflects the fact that the various lowest thresholds are relative high and widely separated, e.g. ${ }_{\Lambda}^{12} \mathrm{~B}+\Lambda$ at $\mathrm{B}_{\Xi}=23.9 \mathrm{MeV}$ followed by ${ }_{\Lambda}^{11} \mathrm{~B}+\mathrm{n}+\Lambda$ at $11.3 \mathrm{MeV}$. Twin-hypernuclei are only produced for $\Xi^{-}$binding energies below the threshold for ${ }_{\Lambda}^{8} \mathrm{Li}+{ }_{\Lambda}^{5} \mathrm{He}$ with $\mathrm{B}_{\Xi}=13.6 \mathrm{MeV}$. As discussed above the frequent observation of twin-hypernuclei [39, 40, 41, 42, 38, 43] signals a conversion from a $\Xi$ state with only moderate binding energy. In this range of $B_{\Xi}$ the production probability of double hypernuclei is comparable to previous estimates within a canonical statistical model [23, [24]. It is important to stress that these numbers do not include a possible pre-equilibrium emission of hyperons during the capture and conversion stage. With respect to the number of stopped $\Xi^{-}$hyperons, pre-equilibrium processes will decrease the yield of double hypernuclei relative to the yield for single hypernuclei (see e.g. [D7]). Indeed in the simulations for the planned PANDA experiment [34] a joint capture $\times$ conversion probability of $5 \%$ was assumed to mimic this pre-equilibrium stage.

Using different $\Xi$-absorbing stable secondary target nuclei ${ }^{9} \mathrm{Be},{ }^{10} \mathrm{~B},{ }^{11} \mathrm{~B},{ }^{12} \mathrm{C}$ and ${ }^{13} \mathrm{C}$ one finds that different double hypernuclei dominate for each target [B]]. Thus combining the information shown in Fig. $\square$ with the measurement of two pion momenta from the subsequent weak decays a unique assignment of various newly observed $\gamma$-transitions to specific double hypernuclei seems possible as intended by the PANDA collaboration [B2], B4] . 


\subsection{The E906 Puzzle}

In 2001 the BNL experiment E906 reported the observation of the ${ }_{\Lambda \Lambda}^{4} \mathrm{H}$ hypernucleus by measuring the sequential pionic decays after a $\left(\mathrm{K}^{-}, \mathrm{K}^{+}\right)$reaction deposited two units of strangeness in a ${ }^{9} \mathrm{Be}$ target [44] (see Fig. (1)). Two structures in the correlated $\pi^{-}$momenta at $(133,114) \mathrm{MeV} / c$ and at $(114,104) \mathrm{MeV} / c$ were observed. The first structure was interpreted as the production of ${ }_{\Lambda}^{3} \mathrm{H}+{ }_{\Lambda}^{4} \mathrm{H}$ twins while the bump at $(114,104) \mathrm{MeV} / c$ was attributed to pionic decays of the double hypernucleus ${ }_{\Lambda \Lambda} \mathrm{H}$. However, as it was pointed out by Kumagai-Fuse and Okabe also twin $\Lambda$-hypernuclear decays of ${ }_{\Lambda}^{3} \mathrm{H}$ and ${ }_{\Lambda}^{6} \mathrm{He}$ are a possible candidate to form this peak if excited resonance states of ${ }^{6} \mathrm{Li}$ are considered [45]. More recently Randeniya and Hungerford showed that the published E906 data can be reproduced without the inclusion of ${ }_{\Lambda \Lambda}^{4} \mathrm{H}$ decay and that it is more likely that the decay of ${ }_{\Lambda \Lambda}^{7} \mathrm{He}$ was observed in the E906 experiment [46]. In their analysis this double hypernucleus was accompanied by a background of coincident decays of single hypernuclei pairs ${ }_{\Lambda}^{3} \mathrm{H}+{ }_{\Lambda}^{4} \mathrm{H},{ }_{\Lambda}^{3} \mathrm{H}+{ }_{\Lambda}^{3} \mathrm{H}$, and ${ }_{\Lambda}^{4} \mathrm{H}+{ }_{\Lambda}^{4} \mathrm{H}$, respectively.

Fig. 1 shows the predicted relative probabilities for the production of particle stable twin and double hypernuclei in the E906 experiment after the capture and conversion of a stopped $\Xi^{-}$ in a secondary ${ }^{9} \mathrm{Be}$ target $\Xi^{-}+{ }^{9} \mathrm{Be} \rightarrow{ }_{\Lambda \Lambda}^{10} \mathrm{Li}^{*}$. As before a $\Xi^{-}$binding energy of $0.5 \mathrm{MeV}$ seems reasonable corresponding to an ${ }_{\Lambda \Lambda}^{10} \mathrm{Li}$ excitation of about $29 \mathrm{MeV}$. The produced yields for this excitation energy are shown in Fig. [3. Here, ground state and excited state(s) - if they exist - have been added and the pion momenta for groundstate decays are assumed. Note, that the production of ${ }_{\Lambda}^{4} \mathrm{H}+{ }_{\Lambda}^{4} \mathrm{H}$ twins is even at an excitation energy of $35 \mathrm{MeV}$ energetically not possible and - unlike to a canonical calculations [24] - does therefore not occur in our micro-canonical model.

Let us first discuss the structure at $(114,104) \mathrm{MeV} / c$ which has been attributed to double hypernuclei decays. Generally the production of double hypernuclei is energetically favored over the production of twins: all possible channels with twin-production lie energetically significantly above the thresholds for ${ }_{\Lambda \Lambda}^{9} \mathrm{Li},{ }_{\Lambda \Lambda}^{7} \mathrm{He}$ and ${ }_{\Lambda \Lambda}^{6} \mathrm{He}$ production in case of the ${ }_{\Lambda \Lambda}^{10} \mathrm{Li}$ compound picture. Correspondingly the production of ${ }_{\Lambda}^{3} \mathrm{H}$ and ${ }_{\Lambda}^{6} \mathrm{He}$ twins which has been suggested as a possible source of the peak structure around $(114,104) \mathrm{MeV} / c$ [45], is in our model by a factor of 30 lower than the ${ }_{\Lambda \Lambda}^{7}$ He production probability. Unlike in the canonical model of Ref. [24] the ${ }_{\Lambda \Lambda}^{7}$ He probability exceeds also the one of a ${ }_{\Lambda \Lambda}^{4} \mathrm{H}$ by more than two orders of magnitude and the production of ${ }_{\Lambda \Lambda}^{5} \mathrm{H}$ by a factor of about 17 in our model. Of course, a direct comparison with the E906 data requires a detailed consideration of the branching ratios for pionic two-body decays many of which are not known so far. Keeping that caveat in mind our microcanonical model supports independently on the assumed production scheme the interpretation of the E906 observation by Randeniya and Hungerford [46] in terms of ${ }_{\Lambda \Lambda}^{7} \mathrm{He}$ decays. Decays from the ground or even exited states of ${ }_{\Lambda \Lambda}^{8} \mathrm{Li}$ or ${ }_{\Lambda \Lambda}^{9} \mathrm{Li}$ can possibly contribute some background to the structure at $(114,104) \mathrm{MeV} / c$.

In order to describe the $(133,114) \mathrm{MeV} / c$ structure of the E906 experiment, the production of ${ }_{\Lambda}^{3} \mathrm{H}+{ }_{\Lambda}^{4} \mathrm{H}$ twins seems mandatory [44]. However, the ${ }_{\Lambda}^{3} \mathrm{H}+{ }_{\Lambda}^{4} \mathrm{H}+\mathrm{t}$ mass lies above the initial mass $m_{0}=m\left(\Xi^{-}\right)+m\left({ }^{9} \mathrm{Be}\right)$ and can therefore not be produced in the $\Xi^{-}+{ }^{9} \mathrm{Be}$ compound production scheme (see right part of Fig. 四). With an energy of $12.6 \mathrm{MeV}$ below $m_{0}$, the channel ${ }_{\Lambda}^{4} \mathrm{H}+{ }_{\Lambda}^{6} \mathrm{He}$ is the most likely twin in the present scenario, followed by the ${ }_{\Lambda}^{4} \mathrm{H}+{ }_{\Lambda}^{5} \mathrm{He}+\mathrm{n}$ decay. Given however the experimental precision of about $1 \mathrm{MeV} / c$ for the momentum calibration in E906 [44], neither the decays of ${ }_{\Lambda}^{4} \mathrm{H}+{ }_{\Lambda}^{6} \mathrm{He}$ with $(133,108) \mathrm{MeV} / c$ nor decays of ${ }_{\Lambda}^{4} \mathrm{H}+{ }_{\Lambda}^{5} \mathrm{He}$ pairs with $(133,99) \mathrm{MeV} / c$ 


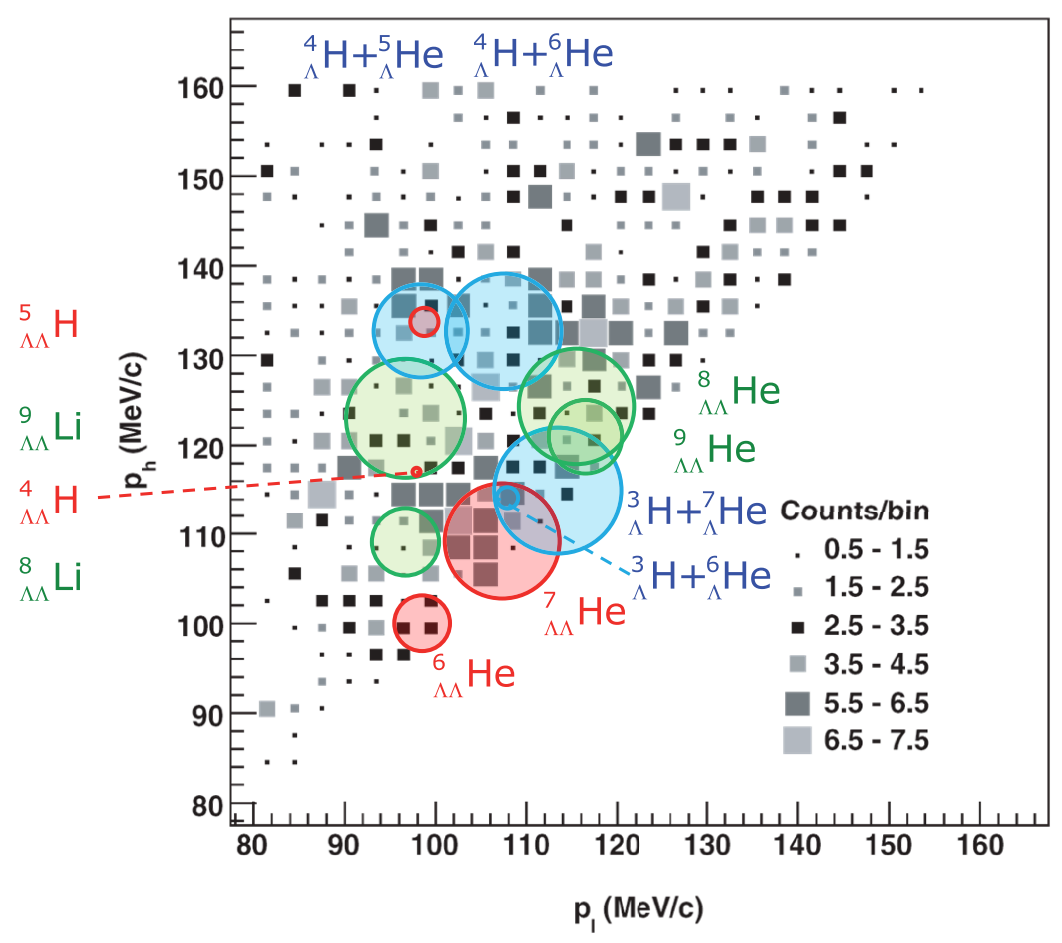

Figure 3: Momenta of two correlated pions measured by the E906 collaboration (grey squares). Overlaid are production yields of various twin pairs (blue), double hypernuclei including their excited states (green) and double hypernuclei with a stable groundstate only (red). The areas of the circles are proportional to the production yields predicted by the statistical model. Pionic decay probabilities are not included in this plot.

seem to explain the structure around $(133,114) \mathrm{MeV} / c$. Of course in particular the first one could contribute to this enhancement in the tail region. Even more intriguing is however the fact that the statistical models predict a production of ${ }_{\Lambda}^{4} \mathrm{H}+{ }_{\Lambda}^{4} \mathrm{H}$ twins exceeding the ${ }_{\Lambda}^{3} \mathrm{H}+{ }_{\Lambda}^{4} \mathrm{H}$ production. Considering furthermore the branching ratios for two-body $\pi^{-}$decays of $\Gamma_{\pi^{-}+{ }^{3} \mathrm{He}} / \Gamma_{\text {total }} \approx 0.26$ [47] and $\Gamma_{\pi^{-}+{ }^{4} H e} / \Gamma_{\text {total }} \approx 0.5$ [48] the absence of a bump which could be attributed to ${ }_{\Lambda}^{4} \mathrm{H}+{ }_{\Lambda}^{4} \mathrm{H}$ is particularly puzzling. A similar conclusion is obtained [35] within the quasi-free/rescattering picture of Yamamoto et al. [24] resulting in the production of excited ${ }_{\Lambda \Lambda}^{8} \mathrm{He}$ or ${ }_{\Lambda \Lambda}^{8} \mathrm{H}$ nuclei.

At first sight it seems that an alternative production process than the ones discussed so far is required to explain the singular structure at $(133,114) \mathrm{MeV} / \mathrm{c}$ in terms of ${ }_{\Lambda}^{3} \mathrm{H}+{ }_{\Lambda}^{4} \mathrm{H}$ twins. Note however that in the initial analysis of the E906 data the bump at $(133,114) \mathrm{MeV} / c$ served as a calibration point for the pion momenta, taking the decay of ${ }_{\Lambda}^{3} \mathrm{H}+{ }_{\Lambda}^{4} \mathrm{H}$ twins as granted [44, 49]. If that structure were indeed caused by ${ }_{\Lambda}^{4} \mathrm{H}+{ }_{\Lambda}^{6} \mathrm{He}$ twins with $(133,108) \mathrm{MeV} / c$, it would of course influence the momentum scale in the region of the $(114,104) \mathrm{MeV} / c$ bump. This bump would then be shifted to approximately $(108,97) \mathrm{MeV} / c$. Considering the uncertainty of $\Delta B_{\Lambda \Lambda}$ also such a momentum scale would be compatible with the decay of ${ }_{\Lambda \Lambda}^{7} \mathrm{He}$ and ${ }_{\Lambda \Lambda}^{8} \mathrm{Li}$ or a mixture of both. The absence of ${ }_{\Lambda \Lambda}^{9}$ Li nuclei may be related to the decreasing pionic decay probability with increasing nuclear mass. Clearly, the present statistical decay model needs to be complemented by quantitative weak decay calculations (see e.g. Ref. [50]]) to further corroborate our conjecture. 


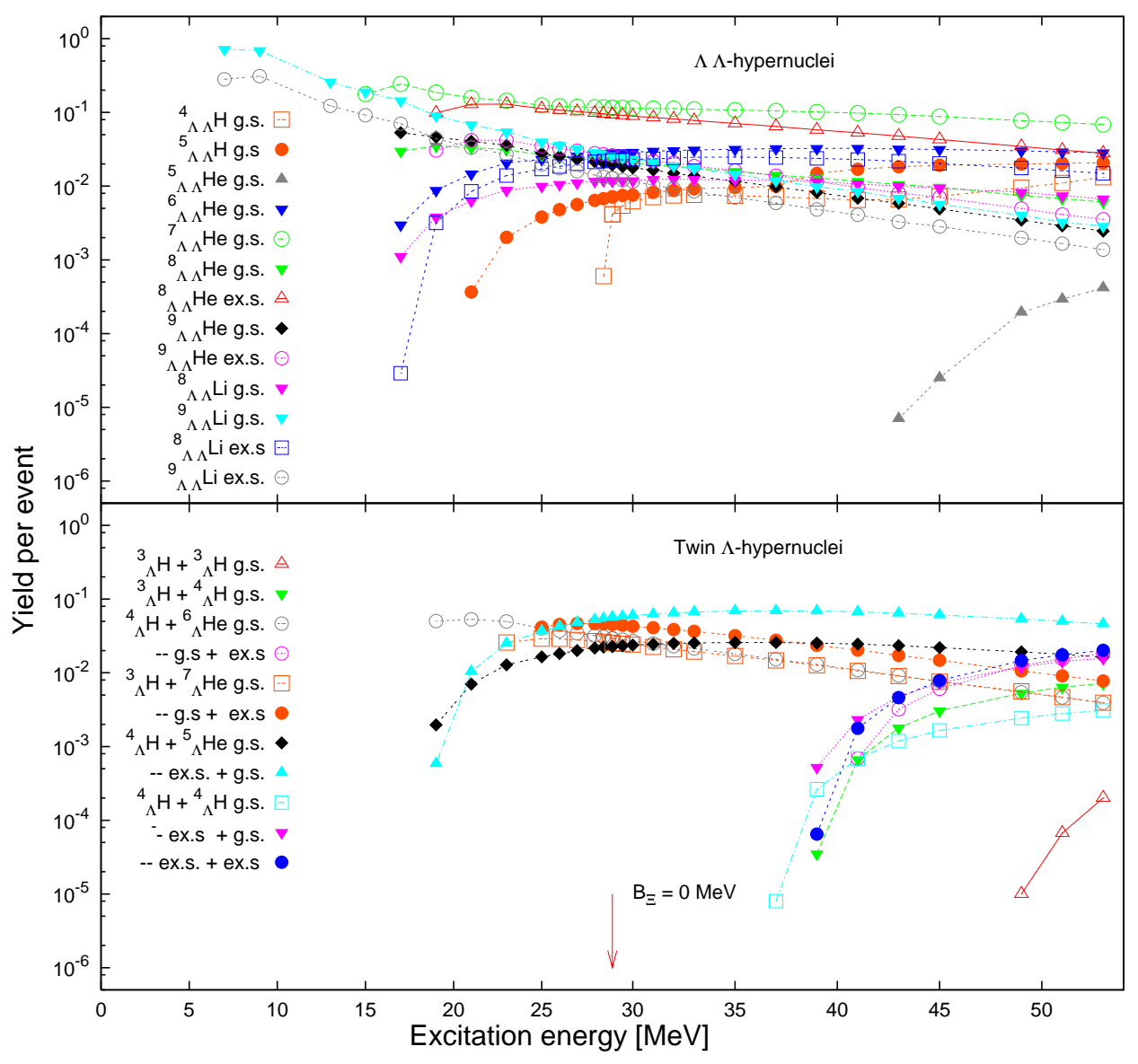

Figure 4: Relative production probability of double (top part) and twin hypernuclei (lower part) for a primary ${ }_{\Lambda \Lambda}^{10} \mathrm{Li}$ nucleus as a function of its excitation energy.

\section{Antihyperons in Nuclei}

Concerning antibaryons, realiable information on their nuclear potential are available only for antiprotons. Antihyperons annihilate quickly in normal nuclei and spectroscopic information is therefore not directly accessible. Mishustin recently suggested to study deeply bound antibaryonic nuclei via various characteristic signals in their decay process [ [1], 52]. It is however not obvious whether these proposed observables will provide unique and quantitative signals of deeply bound antihyperonic systems.

Quantitative information on the antihyperon potentials relative to that of the corresponding hyperon may be obtained via exclusive antihyperon-hyperon pair production close to threshold in antiproton- nucleus interactions [53]. Once these hyperons leave the nucleus and are detected, their asymptotic momentum distributions will reflect the depth of the respective potentials. However, since in the $\bar{p}$ center-of-mass the distribution of the produced baryon-antibaryon pair will usually not be isotropic, the analysis can rely only on the transverse momenta of the outgoing baryons. In Ref. [53] it was demonstrated that the individual transverse momentum distributions alone do not 
allow to extract unambiguous information on the potential of antihyperons. Instead, the transverse momentum asymmetry $\alpha_{T}$ defined in terms of the transverse momenta of the coincident particles can be used to explore event-by-event correlations:

$$
\alpha_{T}=\frac{p_{T}(\Lambda)-p_{T}(\bar{\Lambda})}{p_{T}(\Lambda)+p_{T}(\bar{\Lambda})}
$$

In a purely classical, non-relativistic picture this asymmetry is of the order of $\Delta U / 4 \cdot E_{0}$, where $\Delta U$ is the potential difference and $E_{0}$ the typical kinetic energy of the hadrons.

\subsection{Antihyperon-Hyperon Pair Production and Propagation in Nuclei}

In Ref. [53] we have examined the influence of the potentials on the transverse momentum asymmetry of coincident hyperons and antihyperons by means of a schematic Monte Carlo simulation. Albeit crude, this classical approach allows to explore the role of different features of the reaction in a transparent way.

The absorption of the antiprotons entering the target nucleus determines the points of annihilation inside the nucleus and the paths which the eventually produced hyperons and antihyperons have to pass inside the nucleus prior to emission. Because of the strong absorption of the antihyperons, the emitted hyperon-antihyperon pairs are - unlike in inclusive reactions [54, [55] - created close to the corona of the target nucleus at a typical density of 20 to $25 \%$ of the central nuclear density. In reactions close to threshold the Fermi motion of the protons inside the nuclear target contributes significantly to the final momenta. Lacking any detailed experimental information it is assumed that the annihilation cross sections for antihyperons show a similar momentum dependence as the $\overline{\mathrm{p} p}$ system [15, 56].

The energy and the momentum of the baryons propagating within the nucleus are related according to [5]]:

$$
(E-V)^{2}=\left(M_{0}+S\right)^{2}+\mathbf{P}_{\mathbf{i n}}^{2}
$$

Here $V$ and $S$ denote the real part of the vector and scalar potential, respectively. The relation between the momenta inside and outside of the nuclear potential are approximated by

$$
\mathbf{P}_{\text {out }}{ }^{2}+M_{0}^{2}=\left(\sqrt{\left(M_{0}+S\right)^{2}+\mathbf{P}_{\text {in }}^{2}}+V\right)^{2} .
$$

Refractive effects at the potential boundary were ignored. The default parameters for the scalar and vector potentials of the various baryons at normal nuclear density $\rho_{0}$ were adopted from Ref. [58]. Since the antiproton annihilation and the subsequent antihadron-hadron pair production take place in the nuclear periphery at low densities $\rho$ we assumed a linear density dependence $\propto \rho / \rho_{0}$ for all vector and scalar potentials.

\subsection{Transverse Momentum Correlations at $\overline{\mathrm{P}} \mathrm{ANDA}$}

For a compact representation of event-by-event correlations we examine the transverse momentum asymmetry $\alpha_{T}$ as a function of the longitudinal asymmetry $\alpha_{L}$, where $\alpha_{L}$ is defined by analogy to Eq. $]$ in terms of the longitudinal momentum components:

$$
\alpha_{L}=\frac{p_{L}(\Lambda)-p_{L}(\bar{\Lambda})}{p_{L}(\Lambda)+p_{L}(\bar{\Lambda})}
$$




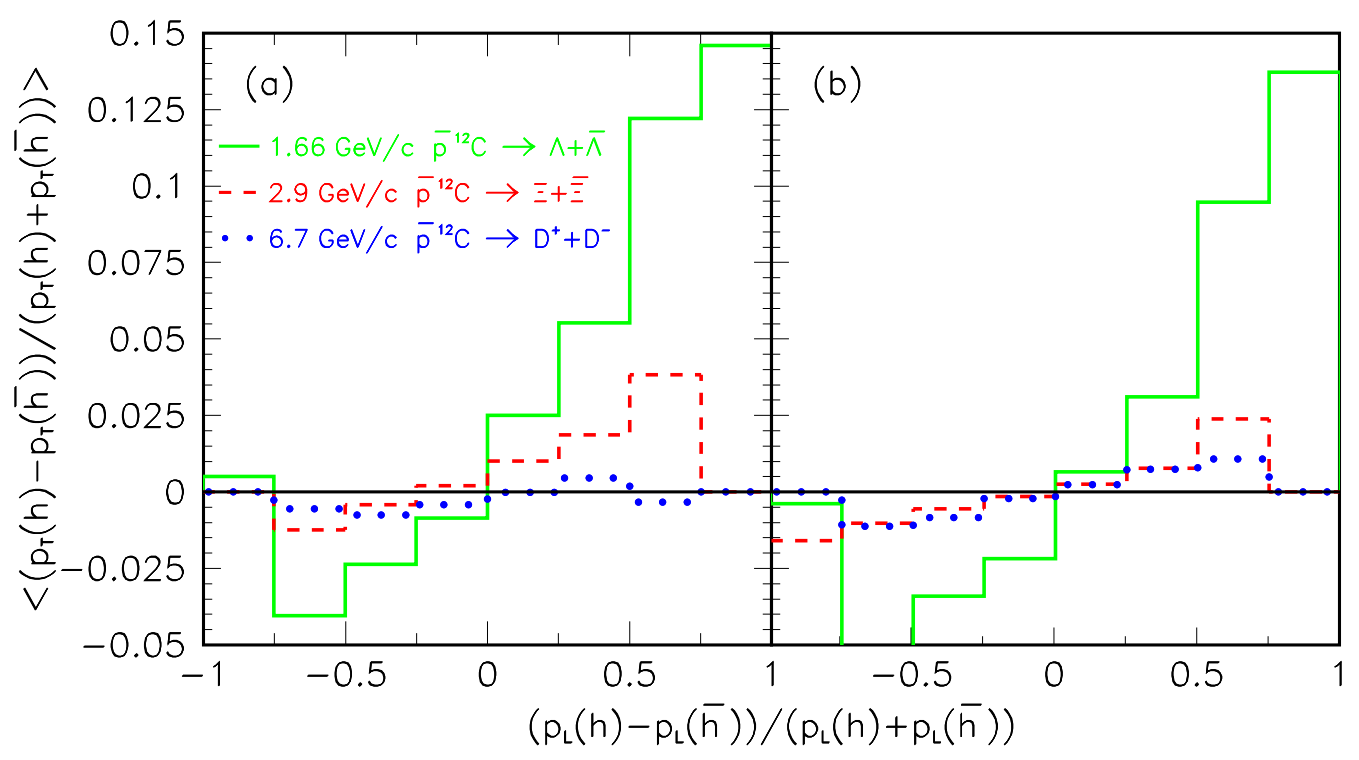

Figure 5: Left part: Average transverse momentum asymmetry of $\Lambda \bar{\Lambda}$ (solid line), $\Xi \bar{\Xi}$ (dashed line) and $\mathrm{D}^{+} \mathrm{D}^{-}$pairs (dotted line) produced exclusively in $1.66,2.9$ and $6.7 \mathrm{GeV} / c \overline{\mathrm{p}}^{12} \mathrm{C}$ interactions, respectively. Right part: the same as in part (a) but taking the momentum dependence of the potential into account.

In future more sophisticated analysis methods which e.g. use mixed events as a reference, further details of these correlations may be revealed.

Transverse momentum correlations can in principle be analyzed for each hadron-antihadron pair produced exclusively in $\bar{p} A$ interactions. As examples, the solid, dashed and dotted histograms in figure Бla show the average transverse momentum asymmetries of $\Lambda \bar{\Lambda}, \Xi \bar{\Xi}$ and $\mathrm{D}^{+} \mathrm{D}^{-}$pairs produced in 1.66, 2.9 and $6.7 \mathrm{GeV} / c \overline{\mathrm{p}}+{ }^{12} \mathrm{C}$ interactions, respectively. For simplicity, isotropic center-of-mass distributions were assumed in case of the $\Xi \bar{\Xi}$ and $\mathrm{D}^{+} \mathrm{D}^{-}$production. For the $\Xi$ and $\bar{\Xi}$ baryons the same absorption cross sections as for the $\Lambda$ and $\bar{\Lambda}$ were adopted, whereas for $\mathrm{D}^{-}$ and $\mathrm{D}^{+}$mesons energy independent absorption cross sections of 10 and $90 \mathrm{mb}$, respectively, were taken. In the left part of Fig. 1 no momentum dependence of the potentials was considered.

In line with the classical picture mentioned above $\left(\alpha_{T} \propto \Delta U / 4 \cdot E_{0}\right)$ the smaller asymmetries for the heavier particles are a consequence of the the large $\Xi$ hyperon and D meson laboratory momenta. In the simulations of Fig. \b the momentum dependence of the potentials was added by means of the scaling factor [5]]. As expected the transverse asymmetry is shifted towards more negative values. Nevertheless the asymmetry remains sizable even for the $\bar{\Xi} \bar{\Xi}$ case. At PANDA when reaching the design luminosity a measurement of $\alpha_{T}$ for $\Xi \bar{\Xi}$ pairs with a precision of about $10 \%$ will require a measurement time of typically less than a day. Furthermore it puts only moderate constraints on the detector performance, e.g. the tracking capabilities of the central vertex detector.

The fact that energy and momentum conservation are the main ingredients of the proposed method raises hope that similar results might be obtained by more sophisticated calculations. Since most of the emitted hyperon-antihyperon pairs are created in the nuclear periphery at subsaturation density, a neutron skin of neutron rich target nuclei may help to explore different effective potentials. Possible deflections at the potential boundary which are ignored in the present work may be 
at least partly eliminated by demanding that the target nucleus remains intact. Furthermore, it may be interesting to study questions related to the formation time by using target nuclei of different size. Finally, it should be noted that this method can be extended to the case of photo- or even electro-production of short-lived resonances in nuclei decaying into particle-antiparticle pairs.

This work was supported by the BMBF under Contract numbers 06MZ225I and 06MZ9182. A.S.L. acknowledges the support from the State of Rhineland-Palatinate via the Research Centre 'Elementary Forces and Mathematical Foundations' (EMG). We also thank the European CommunityResearch Infrastructure Integrating Activity Study of Strongly Interacting Matter (HadronPhysics2, Grant Agreement n. 227431; SPHERE network) under the Seventh Framework Programme of EU for their support.

\section{References}

[1] T.D. Lee and C.N. Yang, Nuovo Cim. 3, 749 (1956).

[2] H.-P. Dürr and E. Teller, Phys. Rev. 101, 494 (1956); H.-P. Dürr, Phys. Rev. 103, 469 (1956).

[3] Hans-Peter Dürr, Phys. Rev. 109, 1347 (1958).

[4] G. Goldhaber and J. Sandweiss, Phys. Rev. 110, 1476 (1958).

[5] G. Bendiscioli and D. Kharzeev, Rivista del Nuovo Cimento 17, 1 (1994).

[6] P. D. Barnes et al., Phys. Rev. Lett. 29, 1132 (1972).

[7] G. Backenstoss et al., Physics Letters B 41, 552 (1972).

[8] P. Roberson et al., Phys. Rev C 16, 1945 (1977).

[9] H. Poth et al., Nucl. Phys. A 294, 435 (1978).

[10] E. Friedman and A. Gal, Phys. Rep. 452, 89, (2007).

[11] E. H. Auerbach, C. B. Dover, and S. H. Kahana, Phys. Rev. Lett. 46, 702 (1981).

[12] C.J. Batty, Nucl. Phys. A 372, 433 (1981).

[13] E. Friedman, A. Gal and J. Mares, Nucl. Phys. A 761, 283 (2005).

[14] Stefan Teis et al., Phys. Rev. C 50, 388 (1994).

[15] C. Spieles et al., Phys. Rev. C 53, 2011 (1996).

[16] A. Sibirtsev et al., Nucl. Phys. A 632, 131 (1998).

[17] C.B. Dover and J.M. Richard, Phys. Rev. C 21, 1466 (1980).

[18] A. Faessler, G. Lübeck and K. Shimizu, Phys. Rev. D 26, 3280 (1982).

[19] A.K.Kerman and M.S. Weiss, Phys. Rev. C 8, 408 (1973).

[20] A.S. Botvina and J. Pochodzalla, Phys. Rev. C 76, 024909 (2007).

[21] The STAR Collaboration, Science 328, 58-62 (2010).

[22] Y. Yamamoto, M. Sano and M. Wakai, Prog. Theor. Phys. Suppl. 117, 265 (1994).

[23] T. Yamada and K. Ikeda, Phys. Rev. C 56, 3216 (1997).

[24] Y. Yamamoto, M. Wakai, T. Motoba, T. Fukuda, Nucl. Phys. A 625, 107 (1997). 
[25] Y. Hirata et al., Nucl. Phys. A 639, 389c (1998).

[26] Y. Hirata et al., Prog. Theor. Phys. 102, 89 (1999).

[27] S. Aoki et al., KEK E176 Collaboration, Nucl. Phys. A 828, 191 (2009).

[28] A. Ohnishi, Y. Hirata, Y. Nara, S. Shinmura and Y. Akaishi, Nucl. Phys. A 684, 595 (2001).

[29] K. Ikeda et al., Prog. Theor. Phys. 91, 747 (1994).

[30] Y. Yamamoto et al., Prog. Theor. Phys. Suppl. 117, 281 (1994).

[31] C.J. Batty, E. Friedman, A. Gal, Phys. Rev. C 59, 295-304(1999).

[32] J. Pochodzalla, Nucl. Instr. Meth B 214, 149 (2004).

[33] Panda Collaboration, Technical Progress Report (GSI Darmstadt), pp. 1-383 (2005).

[34] PANDA Collaboration, Physics Performance Report for PANDA, arXiv:0903.3905.

[35] A. Sanchez Lorente, A. Botvina, J. Pochodzalla, submitted for publication.

[36] E. Fermi, Progr. Theor. Phys. 5, 570 (1950).

[37] C.B. Dover and A. Gal, Ann. Phys. 147, 309 (1983).

[38] S. Aoki et al., Phys. Lett. B 355, 45 (1995).

[39] D.H. Wilkinson, S.J. St. Lorant, D.K. Robinson, and S. Lokanathan, Phys. Rev. Lett. 3, 397 (1959).

[40] P.H. Steinberg and R.J. Prem, Phys. Rev. Lett. 11, 429 (1963).

[41] A. Bechdolff, G. Baumann, J.P. Gerber, and P. Cüer, Phys. Lett. 26B, 174 (1968).

[42] S. Aoki et al., Prog. Theor. Phys. 89, 493 (1993).

[43] A. Ichikawa et al., Phys. Lett. B 500, 37 (2001).

[44] J.K. Ahn et al., Phys. Rev. Lett. 87, 132504-1 (2001).

[45] Izumi Kumagai-Fuse and Shigeto Okabe, Phys. Rev. C 66, 014003 (2002).

[46] S.D. Randeniya and E.V. Hungerford, Phys. Rev. C 76, 064308 (2007).

[47] H. Kamada,J. Golak, K. Miyagawa, H. Witala, W. Glöckle, Phys. Rev. C 57, 1595 (1998).

[48] H. Outa et al., Nucl. Phys. A639, 251c (1998).

[49] Joe P. Nakano, Study of Double-Lambda Hypernuclei by Using Cylindrical Detector System, Ph.D. Thesis, Center for Nuclear Study, University of Tokyo, Report CNS-REP-28 (2000).

[50] A. Gal, Nucl.Phys.A 828, 72 (2009).

[51] I.N. Mishustin et al., Phys. Rev. C 71, 035201 (2005).

[52] A.B. Larionov et al., Phys.Rev.C78, 014604 (2008).

[53] J. Pochodzalla, Phys. Lett. B 669, 306 (2008).

[54] A. Sibirtsev, K. Tsushima, and A.W. Thomas, Eur. Phys.J. A6, 351 (1999).

[55] H. Lenske and P. Kienle, Phys. Lett. B 647, 82 (2007).

[56] H. Weber, E.L. Bratkovskaya, and H. Stöcker, Phys. Rev. C 66, 054903 (2002).

[57] T. Yamazaki and Y. Akaishi, Phys. Lett. B 453, 1 (1999).

[58] K. Saito, K. Tsushima, and A.W. Thomas, Prog.Part.Nucl.Phys. 58, 1 (2007).

[59] C. Gale, G. Bertsch and S. Das Gupta, Phys. Rev C 35, 1666 (1987). 